\title{
Installation effects on an ultrasonic flow meter with implications for self diagnostics
}

\author{
Carl Carlander *, Jerker Delsing \\ Division of Industrial Electronics, Luleå University of Technology, S-971 87 Luleå, Sweden
}

Received 14 June 1999; received in revised form 2 November 1999; accepted 23 December 1999

\begin{abstract}
A small ultrasonic flow meter for water was exposed to five different test configurations, a reference experiment, a single elbow, a double elbow out of plane, a reduction in pipe diameter and a pulsating flow experiment. All tests were performed in a flow calibration facility ranging over Reynolds number from 25 to 110000 . The experiments with the four installation effects were compared with a reference experiment. The error and the change in standard deviation compared to the reference experiment were calculated. The standard deviation serve as a measure of the noise level of the flow meter. The results show that all disturbances generated errors in the flow measurement. The maximum errors were mainly in the range of $2-4 \%$ of flow rate, but at very low flow rates the pulsating flow caused larger errors. In most of the flow range there were no or smaller errors. All installation effects also generated an increase in the noise level. The different pipe configurations increased the standard deviation up to more than $100 \%$. The pulsating flow induced even higher enlargements in the noise level. The errors and the increase in the standard deviation are present in about the same flow ranges. The results demonstrate not only that the installation effects tested introduce errors in the flow measurements but also that these effects can be detected from the noise level in the data. The noise level was determined from the standard deviation. This could be interpreted as that the disturbances amplify the turbulence intensity. Thus the standard deviation can be used as a measure of the turbulence. The presence of a disturbance could be recognised by comparing the magnitude of the noise level in the present data with a reference level valid for the measured flow rate. A procedure like this could possibly be performed by the meter itself in operation. (C) 2000 Elsevier Science Ltd. All rights reserved.
\end{abstract}

Keywords: Ultrasonic flow meter; Installation effects; Pulsating flow; Self diagnostics; Liquid flow measurements

\section{Introduction}

A project concerning measurement quality assurance in district heating systems is in progress at Luleå University of Technology. One of the main desires of the district heating industry is accurate heat transfer measurements. It is well known that the flow measurement involved will be affected by different installation effects. One aspect of the project is to examine the possibility of self diagnostic techniques for flow meters.

Installation effects are regarded as one of the most serious origin of errors in flow measurements. For this reason experimental work concerning installation effects has been performed. All commonly used flow meter types are to different degrees affected by installation

\footnotetext{
* Corresponding author. Tel.: +46-920-91729; fax: +46-920-72082.

E-mail address: carl.carlander@sm.luth.se (C. Carlander).
}

effects. Examples are [1-3]. Ultrasonic flow meters are also affected by different flow disturbances. It has been shown, both with experimental work and simulations, that single and double elbows in front of an ultrasonic flow meter will cause errors [4-7]. Further it has been demonstrated that pulsating flow will give rise to errors $[8,9]$. Work has also been done concerning diagnostic methods for other types of flow meters however not dealing with installation effects but other error sources affecting the performance [10,11].

Previous work considered both static installation effects caused by pipe bends and dynamic effects generated by pumps [9]. The experimental work presented in this paper is a continuation of the work in $[9,14]$. The same experimental data used in this paper was also used in [14]. In [14] only the errors caused by installation effects were considered. In this paper the noise level in the data will also be investigated. Compared to [9] the ultrasonic flow meter tested is a new improved meter 
with higher sampling rate and better precision. Further the test facility where the experiments were carried out provided an increased flow range, with both lower and higher flow rates. In [9] a pulsating flow was generated by using a pump. The frequency of the pulsation generated in these new experiments is lower, better imitating a fast control valve that might be found in district heating subscriber stations.

In this paper an ultrasonic flow meter for water was exposed to five different test configurations, one reference set-up and four disturbances. The disturbances, which were mounted in front of the meter, were a single elbow, a double elbow out of plane, a reduction in diameter and a rotating valve. The experiments were performed in a flow meter calibration facility in the flow range with Reynolds number approximately from 25 to 110000 . All four of the disturbances caused errors when compared with the reference experiment [14].

For the purpose of flow meter self diagnostics this paper will investigate the possibility of using the noise level in the data to detect if the meter was exposed to one of the disturbances. The basic assumption is that the noise level in the signal from the flow meter is well correlated to turbulence intensity in the meter.

\subsection{The ultrasonic flow meter}

The tested flow meter is a small ultrasonic flow meter for water of the sing-around type. This flow meter has a diagonal sound path tilted $20^{\circ}$ compared with the pipe centre line. The distance between the two transducers is $59.5 \mathrm{~mm}$. The diameters of the sound path and the pipe are both $10 \mathrm{~mm}$. Therefore, the ratio between the sound path diameter and pipe diameter equals one. The diameter of the meter was reduced in order to increase the flow velocity through the meter. An initial $19 \mathrm{~mm}$ bore diameter is reduced to $10 \mathrm{~mm}$ by a $10^{\circ}$ cone shaped section. The $25.6 \mathrm{~mm}$ diameter piping of the test facility is connected to the meter body by thread fittings. The meter is also described in [14].

The meter transmitted 150 sound pulses in both the up and the down stream direction. The mean time for the travelling of the sound pulse between the transducers was continuously communicated to a host computer. When a measurement sequence was completed, the host computer calculated the flow rates off line. This arrangement was chosen in order to get a higher sampling frequency of the flow meter. The sampling frequency achieved was $112 \mathrm{~Hz}$. The principle of operation of the flow meter and the algorithms used to calculate the flow velocities are described in [12].

\subsection{The flow meter calibration facility}

The tests were performed in a flow meter calibration facility recently built at Luleå University of Technology.
The calibration facility is based on continuous weighing. This facility is outlined in [13,14].

The flow is generated by a head tank and controlled by control valves. A pulsating flow can be generated by using a rotating butterfly valve. In one of three $11 \mathrm{~m}$ long test runs the experiments were set up. Finally the water was collected in one of three tanks and weighed. By using three scales with different capacities the flow range of the calibration facility was increased. The range is from 0.7 to more than $40000 \mathrm{l} / \mathrm{h}$.

The operation of the facility is controlled by a computer. This computer also collects the data and calculates the flow rate. It also communicates with the host computer of the ultrasonic flow meter.

In Fig. 1 the estimated total uncertainty of the calibration facility with a $95 \%$ confidence is plotted [13].

At flow rates over $20 \mathrm{l} / \mathrm{h}$ the total uncertainty is $\pm 0.1 \%$. The total uncertainty increases at lower flow rates as the precision limit increases. The estimated bias limit is constant with a magnitude of $\pm 0.05 \%$. This performance is satisfactory for the experiments presented in this paper, especially as the bias limit is not of great interest as only the change from the reference experiment is studied in these experiments.

\section{The experiments}

The experiments are intended to imitate flow meter installations that could be found in for example district heating and fresh water distribution systems and small process systems. In these systems for example pipe bends, pumps and fast control valves can be found fairly close to the meter. The flow range tested was from close to zero flow to about $2 \mathrm{~m} / \mathrm{s}$ in $25.6 \mathrm{~mm}$ piping. This flow range is believed to be relevant in the systems mentioned above.

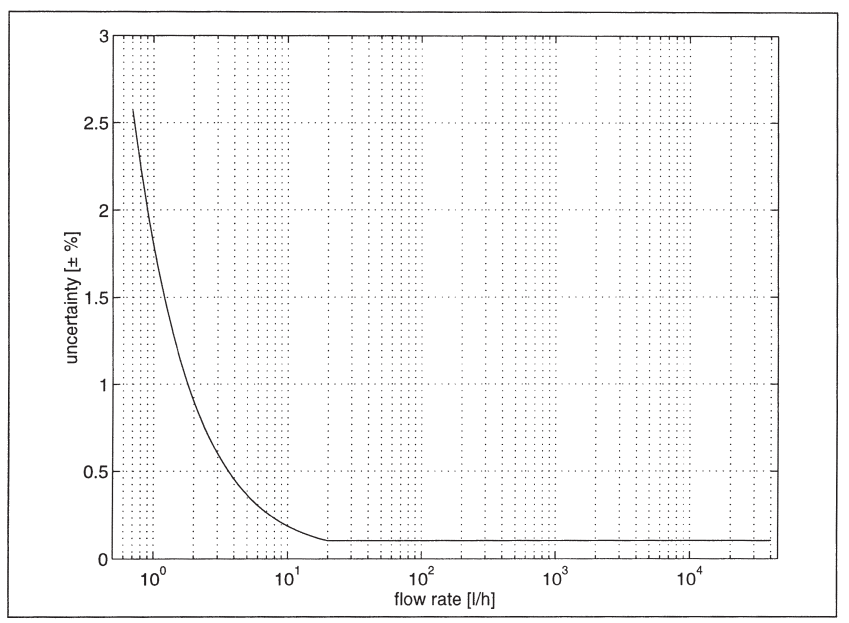

Fig. 1. The estimated total uncertainty as per cent of flow rate for test times of $120 \mathrm{~s}$ [13]. 
The tested ultrasonic flow meter was exposed for five different experimental set-ups:

1. Straight piping was mounted in front of the meter. This worked as a reference experiment.

2. A single elbow was mounted $11 D$ in front of the meter.

3. A double elbow out of plane was mounted $11 \mathrm{D}$ in front of the meter.

4. A diameter reduction was mounted $13 D$ in front of the meter.

5. Finally the meter was exposed to a pulsating flow.

The flow meter was tested over a 1:4400 flow range, with Reynolds number from 25 to 110000 . These correspond to flow velocities of $2.5 \mathrm{~mm} / \mathrm{s}$ to $11 \mathrm{~m} / \mathrm{s}$ in the $10 \mathrm{~mm}$ diameter pipe in the flow meter or 0.7 and 3100 $1 / \mathrm{h}$ in general.

In this range 73 measurements at different flow rates were performed. Each measurement lasted $120 \mathrm{~s}$. This means that, with the $112 \mathrm{~Hz}$ sampling frequency, each of the measuring points consists of a little more than 13000 averaged measurements. The reference experiment was repeated six times and the other experiments three times. It took a little less than $48 \mathrm{~h}$ to acquire all the data in the reference experiment and about $16 \mathrm{~h}$ in the other experiments.

During the experiments the temperature of the water varied freely. The temperature was within $19 \pm 1.5^{\circ} \mathrm{C}$ and corresponds to a change in density of less than $\pm 0.04 \%$. This change was not corrected for.

\section{Results}

The results displaying the errors caused by the disturbances are mainly presented in two different ways by using the meter factor and the error. The meter factor is defined as the ratio between the flow velocity determined by the calibration facility at each measuring point and the mean of the 13000 measurements made by the flow meter.

meter factor $=\frac{v_{\text {calibration }}}{v_{\text {ultrasonic }}}$

The mean meter factor of the six reference experiments was multiplied with the flow measurements made by the flow meter to obtain compensated measurements. This means that the mean of the compensated measurements by the flow meter in the reference experiment will agree with the measurements made by the calibration facility. The results from the following experiments are presented as the percentage error of flow rate, denoted error, compared with the reference experiment. error $=\frac{v-v_{\text {ref,mean }}}{v_{\text {ref,mean }}} 100$

Here $v$ denotes compensated velocity for each point in the measuring sequence of any experiment and $v_{\text {ref,mean }}$ denotes the compensated mean velocity of the six measurements performed at each flow rate in the reference experiment. If the flow meter overestimates the flow compared with the reference experiment, it will show as a positive error. An underestimation will appear as a negative error.

The noise level in the assumed Gaussian distributed signal is represented by the estimated standard deviation based on the about 13000 measurements composing each measuring point. The results concerning the standard deviation are mainly displayed by $\mathrm{std}_{\text {ref,mean }}$ and $\mathrm{std}_{\text {change. }}$. The noise level of the data in the reference experiment is represented by the mean of the six standard deviations estimated at each of the 73 flow rates, denoted $s_{\text {ref,mean }}$. The results from the reference experiment and the experiments with disturbances are presented as the percentage change in the standard deviation from the mean of the reference experiment, $\operatorname{std}_{\text {change }}$

$\operatorname{std}_{\text {change }}=\frac{\text { std }- \text { std }_{\text {ref,mean }}}{\operatorname{std}_{\text {ref,mean }}} 100$

Here std denotes the standard deviation of the measurements in each measuring point of the experiments. If the standard deviation increased in the flow measurements compared with the mean of the reference experiment it will show as a positive change in the plots. If instead the standard deviation decreased a negative change will display.

In the plots Reynolds number was based on the flow determination performed by the calibration facility and the $10 \mathrm{~mm}$ diameter of the ultrasonic flow meter.

\subsection{The reference experiment}

The reference experiment works as base line results. The other experiments with disturbances will be compared with this reference case.

The diameter of the piping in front and after the meter (D) was $25.6 \mathrm{~mm}$. In the reference experiment only straight piping was mounted, $110 \mathrm{D}$ in front of the meter and $48 \mathrm{D}$ behind. This was to ensure a fully developed flow profile at the entrance of the meter.

In Figs. 2 and 3 plots of the mean meter factor curve for the six undisturbed measuring sequences of the reference experiment is presented.

The meter factor is stated as shown in Eq. (1). Fig. 2 presents the mean meter factor of the six reference measurement sequences. Fig. 3 displays the same data as Fig. 2 but with Reynolds number in a logarithmic scale. 


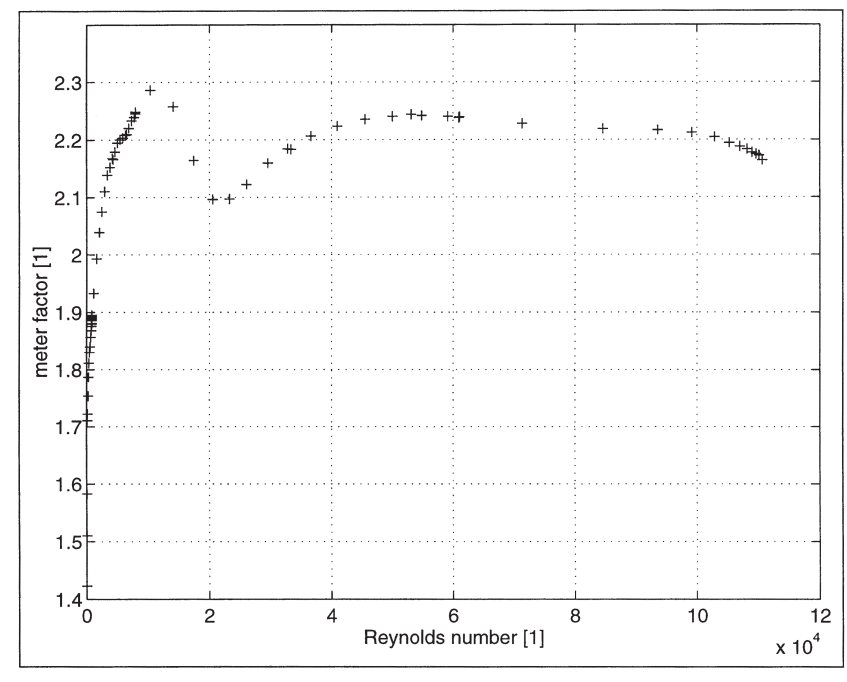

Fig. 2. The mean reference meter factor curve of the six measuring sequences.

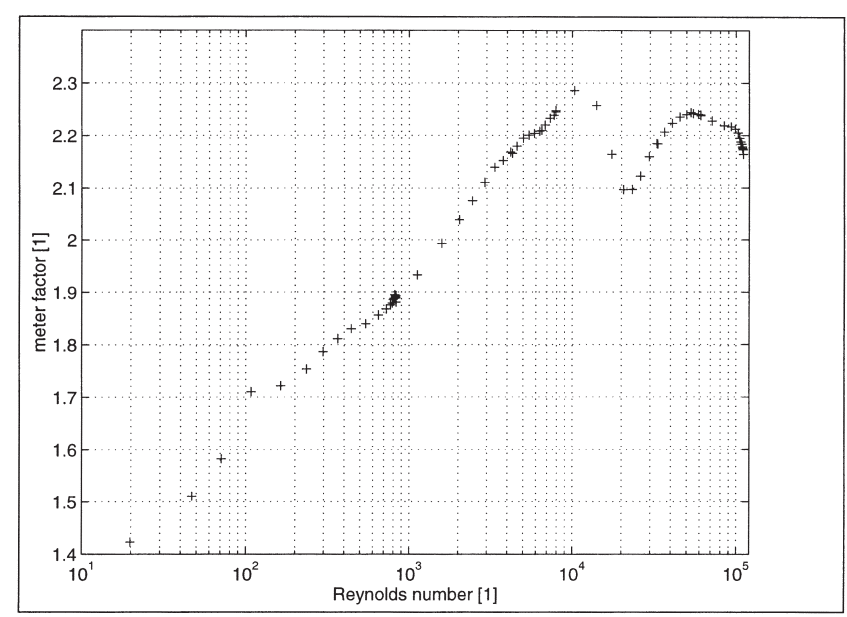

Fig. 3. The mean reference meter factor curve of the six measuring sequences. Reynolds number is in logarithmic scale.

The magnitude of the meter factor for turbulent flow is about 2.2. This could be explained by the geometric design of the meter. This since the sound beam only interacts with the flow slightly less than half the way between the transducers [12]. The distance between the two transducers is $59.5 \mathrm{~mm}$. For a distance of $29 \mathrm{~mm}$ the sound will interact with the flow. For laminar flow the meter factor decreases due to the change to a quadratic flow profile.

The bumps in the meter factor curve at Reynolds number 10000,20000 and 100000 are probably the result of the cavities near the transducers, the fittings of the piping and the reduction in diameter. At Reynolds number 100 an outlier in the third measuring sequence caused a small deviation in the mean meter factor curve. The details of the meter factor curve are not easily understood. It is a complex fluid dynamic problem which probably includes strong recirculation in the cavities and potentially even cavitation. Further investigations are required to fully explain the appearance of the curve.

The compensated mean reference velocity is subsequently subtracted accordingly to Eq. (2) so that the deviation in velocity is displayed in percentage. Fig. 4 displays the error in velocity of each measuring point of the six measuring sequences made with the reference setup compared with the mean. The error in velocity, error, is calculated as shown in Eq. (2).

In Fig. 4 the solid curves indicate the limits confining the data from the six measurements made with a $95 \%$ confidence. These limits represent the repeatability in both the flow meter and the calibration facility. In the following experiment this means that points outside these limits indicate a significant error compared to the reference experiment.

For Reynolds number higher than 3000 the measurements were confined within $\pm 0.5 \%$. For Reynolds number 500,50 and 10 the limits are $\pm 5, \pm 20$ and $\pm 40 \%$. This means that the range with $\pm 0.5 \%$ accuracy is $1: 40$ and the range with $\pm 5 \%$ is $1: 240$. If comparing Fig. 4 with Fig. 1, it can be seen that the uncertainty of the calibration facility will influence the results presented in Fig. 4 only slightly.

In Figs. 5 and 6 the mean standard deviation for the undisturbed reference experiment, $\mathrm{std}_{\text {ref,mean }}$, is presented. Each point in the plots represents a mean of the six measurement sequences made. In Fig. 6 Reynolds number is in logarithmic scale.

The noise level in the measurements increase steadily with higher Reynolds number except for laminar flows. This can be seen in Fig. 6, showing a zoomed plot of the standard deviation.

At Reynolds number below 2000 the standard deviation is almost constant with a magnitude of about 0.3

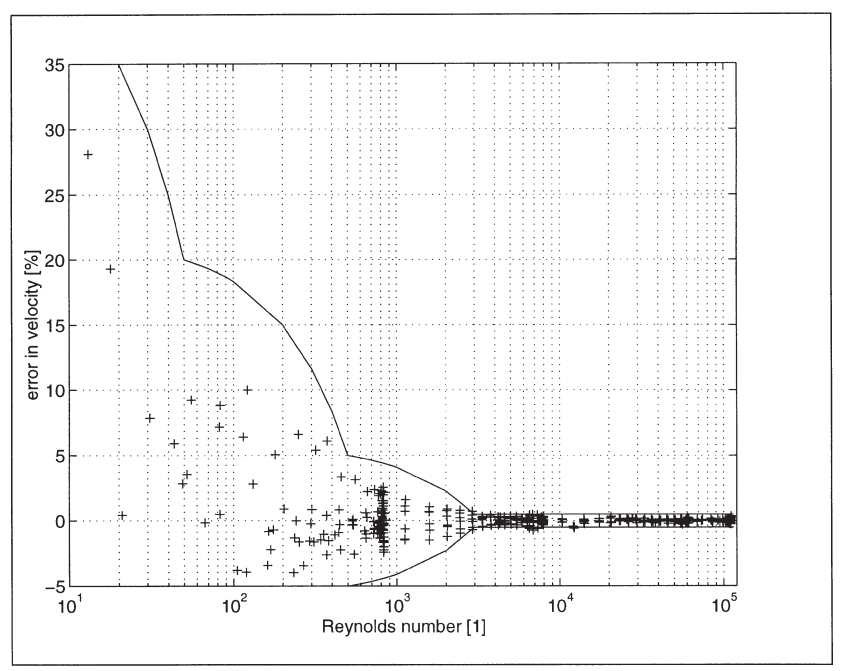

Fig. 4. The percentage error in the six reference measuring sequences. The solid lines represent the limits confining the six reference measurements with a $95 \%$ confidence level. Reynolds number is in logarithmic scale. 


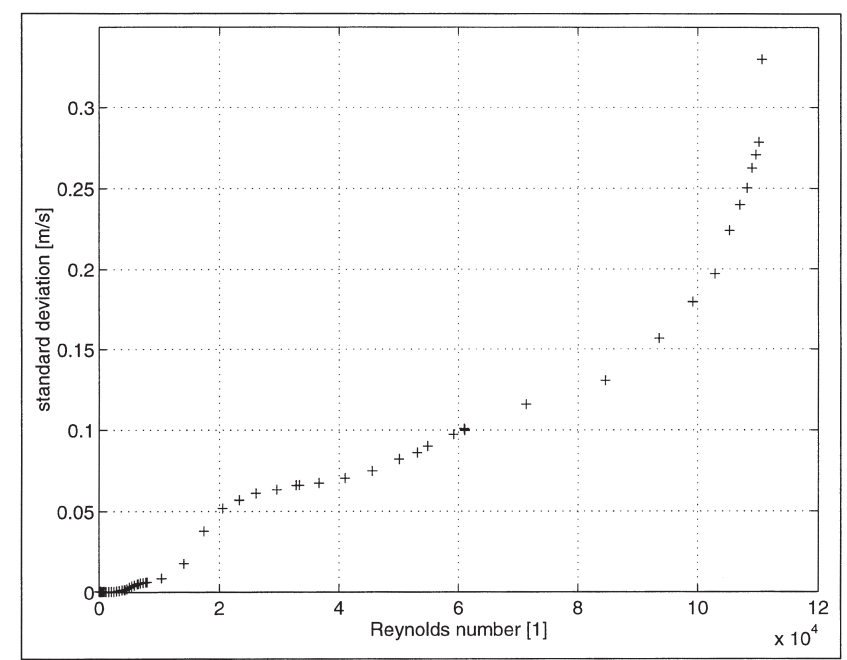

Fig. 5. The mean standard deviation $\operatorname{std}_{\text {ref,mean }}$ of the data in the reference experiment.

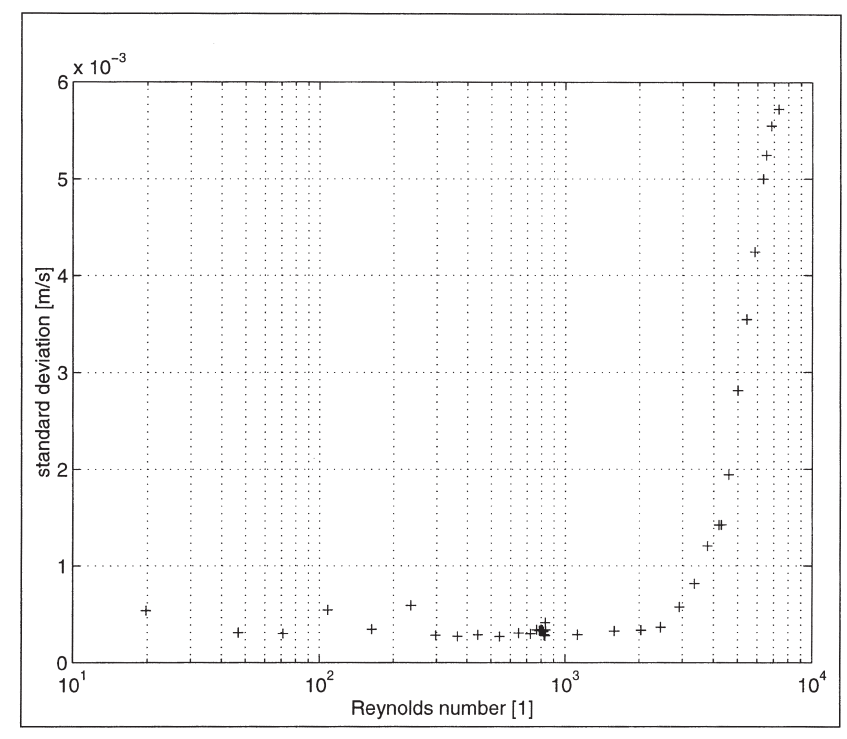

Fig. 6. The mean standard deviation $\operatorname{std}_{\text {ref,mean }}$ of the data in the reference experiment. Reynolds number is in logarithmic scale.

$\mathrm{mm} / \mathrm{s}$. This level represents the basic noise level of the flow meter. At transient flow above Reynolds number of 2000 the noise level in the measurements start to increase as the flow becomes more and more turbulent. As Fig. 5 displays, the standard deviation continues to increase throughout the whole flow range. It seems as if the standard deviation works as a measure of the turbulence intensity present in the flow. The increase of the noise level at Reynolds number over 2000 is not linear. In order to explain the details of the appearance of the curve further investigations are required.

The mean standard deviation shown in Figs. 5 and 6 is subsequently subtracted as shown in Eq. (3) so that the deviation in standard deviation is displayed in per- centage. Fig. 7 presents the change in standard deviation of each measuring point of the six measuring sequences made with the reference set-up compared with the mean. The change in standard deviation, std $_{\text {change }}$, is calculated as shown in Eq. (3).

The solid curves indicate the limits confining all the data from the six measurements made with this reference set-up with a $95 \%$ confidence level. These limits point out the repeatability of the standard deviation estimations made. As with the error this means that points outside these limits in the following experiment indicate a significant change in standard deviation compared to the reference experiment.

Between Reynolds number 15000 and 90000 the measurements are confined within $\pm 5 \%$. For Reynolds numbers from 2000 to 15000 and higher than 90000 the limits are $\pm 20 \%$. At a Reynolds number lower than 2000 the limits increase to $\pm 100 \%$. If comparing Fig. 7 with Fig. 1, it can be seen that the uncertainty of the calibration facility has a negligible influence on the results presented in Fig. 7.

\subsection{The single elbow experiment}

The first installation disturbance tested was the single elbow. There was $11 \mathrm{D}$ of straight piping between the meter and the outlet of the elbow. In front of the elbow a $100 \mathrm{D}$ long straight pipe ensured a fully developed flow profile before the elbow. The bending radius of the elbow equalled the pipe diameter, The angular orientation of the elbow was such that the transducer plane coincided with the elbow plane [14].

In Fig. 8 and in the following plots, $(+)$ marks the first measuring sequence, $(O)$ the second and $(x)$ the third. In

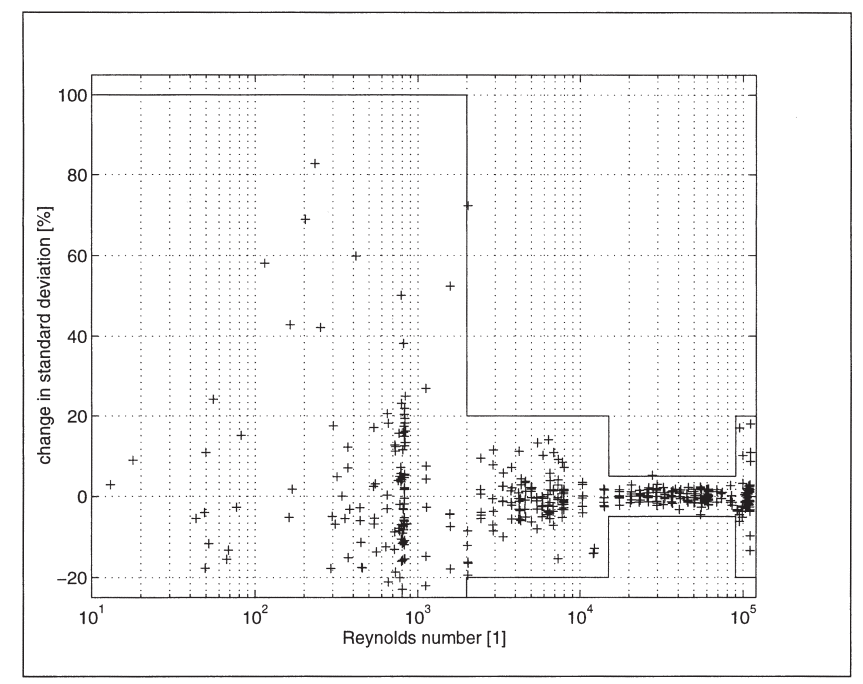

Fig. 7. The change of the standard deviation in per cent $\operatorname{std}_{\text {change }}$ in the reference experiment. The solid lines represent the limits confining all the data from the six measurements with a 95\% confidence level. Reynolds number is in logarithmic scale. 


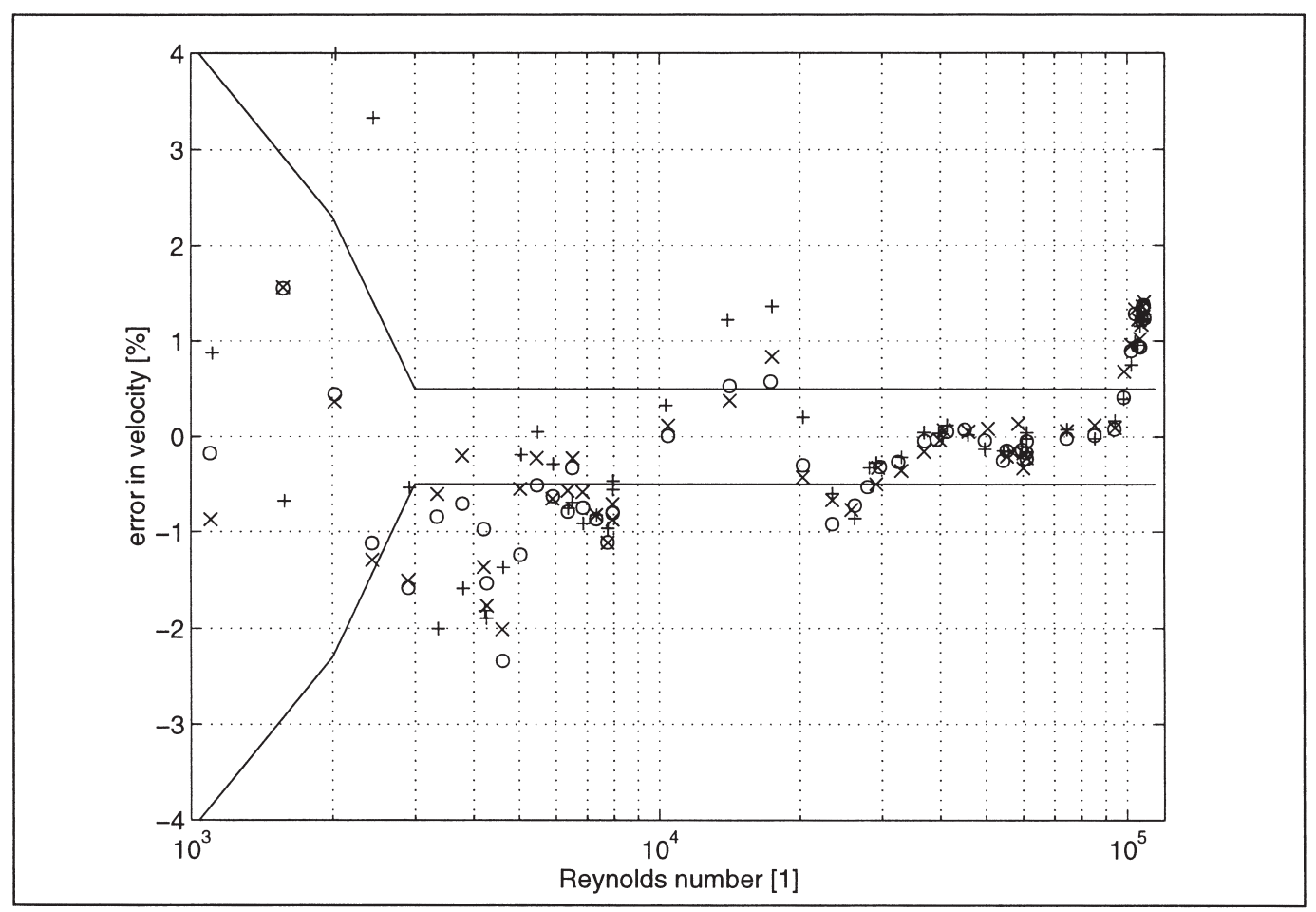

Fig. 8. The percentage error due to the single elbow. The solid lines represent the limits confining the six reference measurements with a $95 \%$ confidence level.

Fig. 8 a plot shows the percentage error in the measured velocity due to the single elbow. On the $\mathrm{x}$-axis Reynolds number are only shown from 1000 to 120000 .

The single elbow causes both over and under estimations in the flow measurements compared to the reference experiment. These errors were significant at Reynolds number in the ranges 2000-30000 and 100000 110 000. The largest error was $3 \%$ and occurred at Reynolds number 4000 . There are no significant difference in the errors for Reynolds number lower than 2000.

The significant errors in Fig. 8 arise at Reynolds numbers where the meter factor curve in Figs. 2 and 3 shows a marked slope. With the single elbow the flow is underestimated when the slope is positive and underestimated when the slope is negative. This shift in the meter factor curve could perhaps be explained by the disturbed flow profile and the triggering of turbulence at lower Reynolds numbers [10]. If adding turbulence to the reference case the data in Figs. 2 and 3 would move left as turbulent behaviour would appear at lower Reynolds numbers. In Fig. 9 a plot of the change in standard deviation due to the single elbow is presented.

The single elbow causes an increase of the standard deviation in the flow measurement in almost the whole flow range. The most distinct increase occurs about in same flow range as the errors show up.

At Reynolds number in the ranges of 4000-8000 and $100000-110000$ the change in standard deviation is between 20 and 100\%. For Reynolds number from
30000 to 90000 the standard deviation increased by about $15 \%$. In this range where were no errors. Below Reynolds number 4000 some measurements show an increased standard deviation but most do not.

The errors and the increase in noise level occur in most cases at the same flow rate. Where there is a significant error there is also an increase in standard deviation. In the range over Reynolds number 20 000-30 000 there is however a small error but not any increase in the noise level. In the range over Reynolds number 30000 90000 there is on the contrary an increase in standard deviation but no error.

The repeatability seems to be about the same as in the reference case, both for the errors and the standard deviation.

\subsection{The double elbow experiment}

The second installation disturbance tested was the double elbow. Between the meter and the outlet of the second elbow there was $11 D$ of straight piping. In front of the first elbow a $100 \mathrm{D}$ long straight pipe ensured again a fully developed flow profile before the elbow. The bending radiuses of both elbows were the same as in the single elbow experiment. The angular orientation of the elbows was such that the angle between the transducer plane and the plane of the elbow closest to the meter was $90^{\circ}$. The two elbows were spaced with $4 \mathrm{D}$ [14]. 


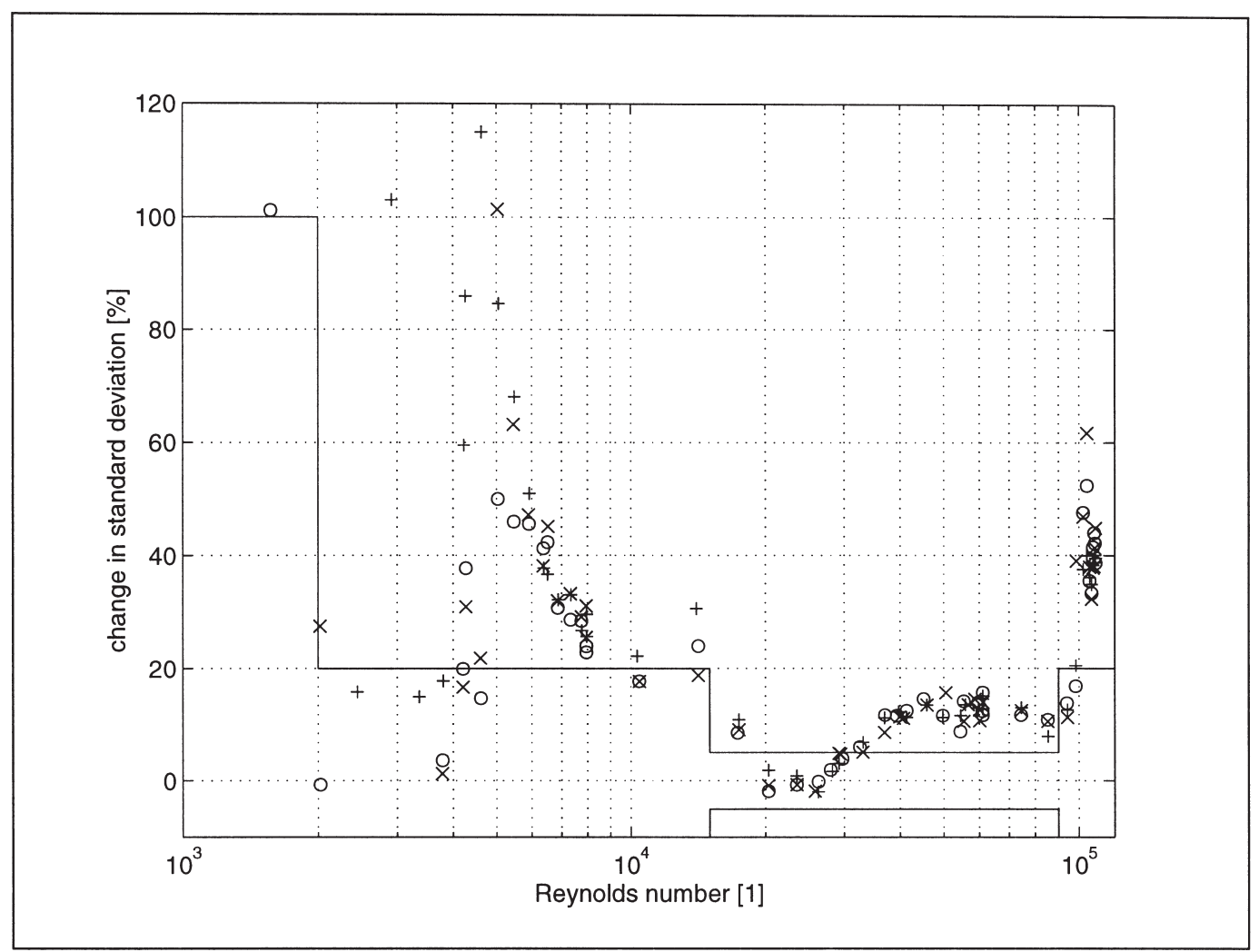

Fig. 9. The change of the standard deviation in per cent $\operatorname{std}_{\text {change }}$ in the single elbow experiment. The solid lines represent the limits confining the six reference measurements with a $95 \%$ confidence level.

Figs. 10 and 11 shows respectively the error in velocity and the change in standard deviation due to the double elbow.

The double elbow caused in general slightly larger errors than the single elbow. The significant errors showed up mainly at Reynolds number in the ranges 3000-8000 and 100 000-110 000. The largest error was about $4 \%$ and occurred at Reynolds number 4000 . Below Reynolds number 3000 all errors are comparable to reference conditions.

The errors caused by the double elbow are similar to those generated by the single elbow. The reason for this could be the spacing of the two elbows [1]. The double and single elbow experiments are however different in the range with Reynolds numbers from 10000 to 40000 . The change of the angular orientation of the elbow closest to the meter could perhaps be the explanation. Earlier investigations on ultrasonic gas flow meters show that the behaviour of the meter can shift when the angular orientation of the elbow is changed [6].

Also the noise level of the data in the double elbow experiment is similar to that in the single elbow experiment. In the ranges of Reynolds number 30 000-60 000 and $100000-110000$ the increase was from 15 to $50 \%$. Also at Reynolds numbers below 20000 the change in standard deviation is almost identical compared to the change that occurred in the single elbow experiment.
The clear increase took place at Reynolds numbers higher than 3000 . The magnitude of the increase is the same as in the single elbow case, $20-100 \%$.

The errors greater than reference conditions and the significant increase in noise level occur also in the double elbow experiment in most cases at the same flow rate. At Reynolds number 20000 and in the range over 50 000-80 000 there is however an increase in standard deviation but no errors.

Again the repeatability was about the same as in the reference experiment. Also in the double elbow experiment the errors and the change in standard deviation mainly took place in the ranges where the meter factor curve inclines.

\subsection{The diameter reduction experiment}

The third installation disturbance tested was the reduction in diameter. The diameter of the piping in front of the meter was reduced by using a $45^{\circ}$ cone shaped pipe segment. The diameter of the pipe before the reduction was $2 D(51.2 \mathrm{~mm})$ and after $1 D(25.6 \mathrm{~mm})$. Between the meter and the outlet of the cone $13 \mathrm{D}$ of straight piping was mounted [14].

Fig. 12 presents the percentage error in velocity due to the diameter reduction.

The errors caused by the reduction in diameter were 


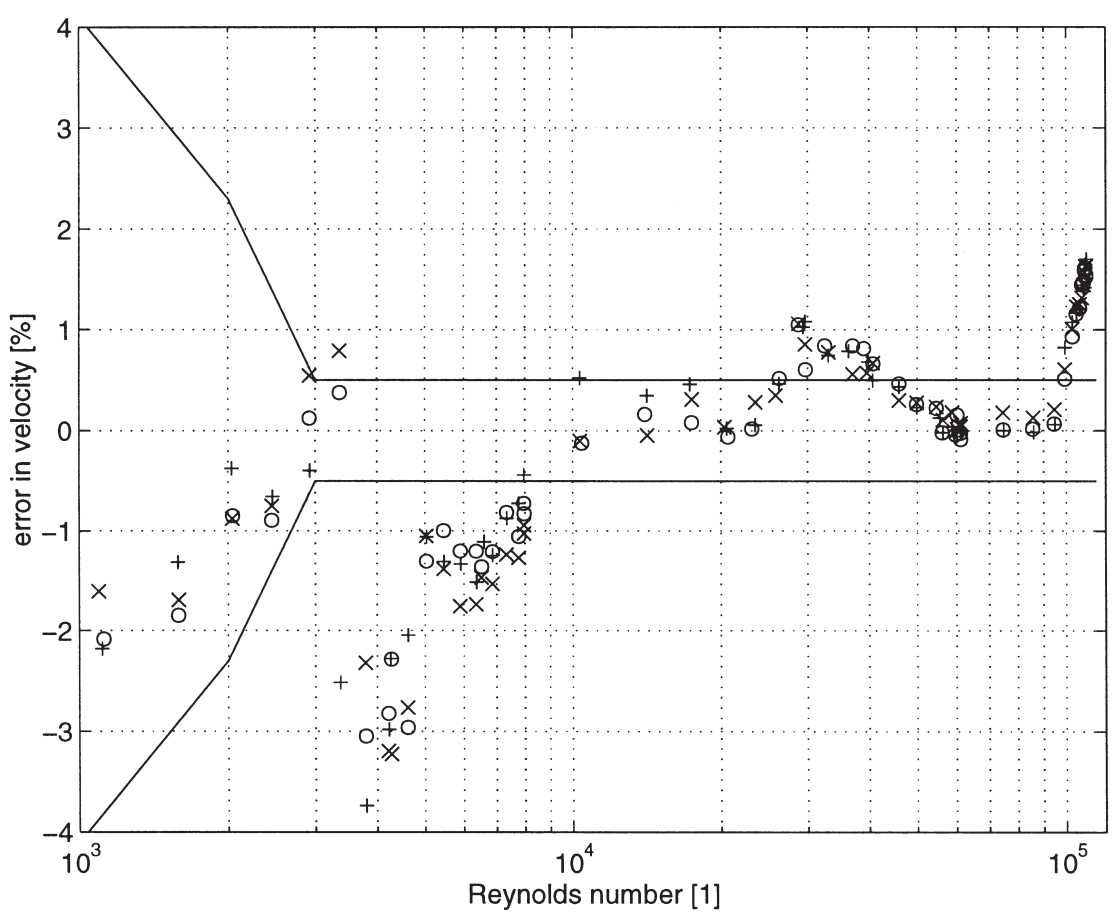

Fig. 10. The percentage error due to the double elbow. The solid lines represent the limits confining the six reference measurements with a $95 \%$ confidence level.

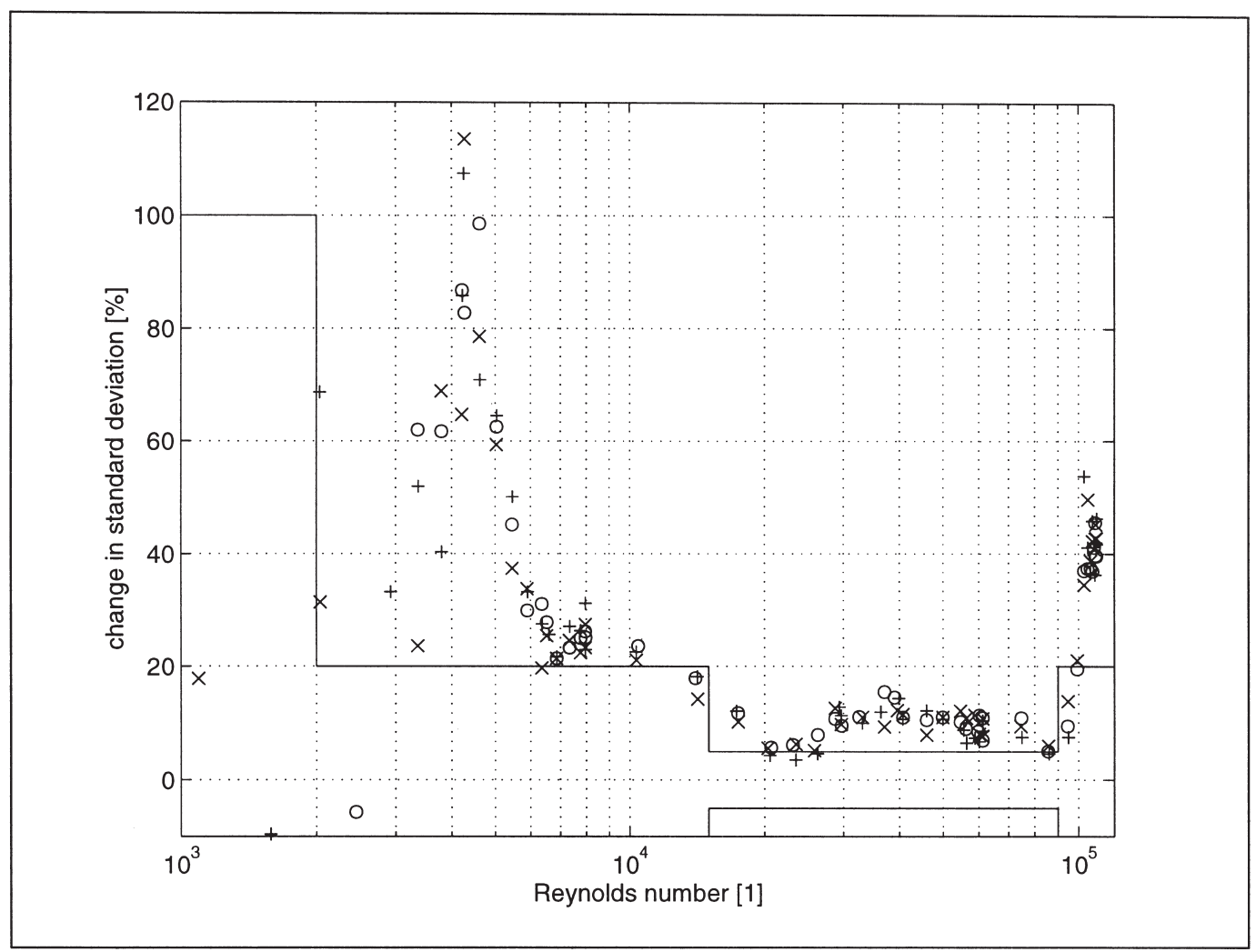

Fig. 11. The change of the standard deviation in per cent $\operatorname{std}_{\text {change }}$ in the double elbow experiment. The solid lines represent the limits confining the six reference measurements with a $95 \%$ confidence level. 


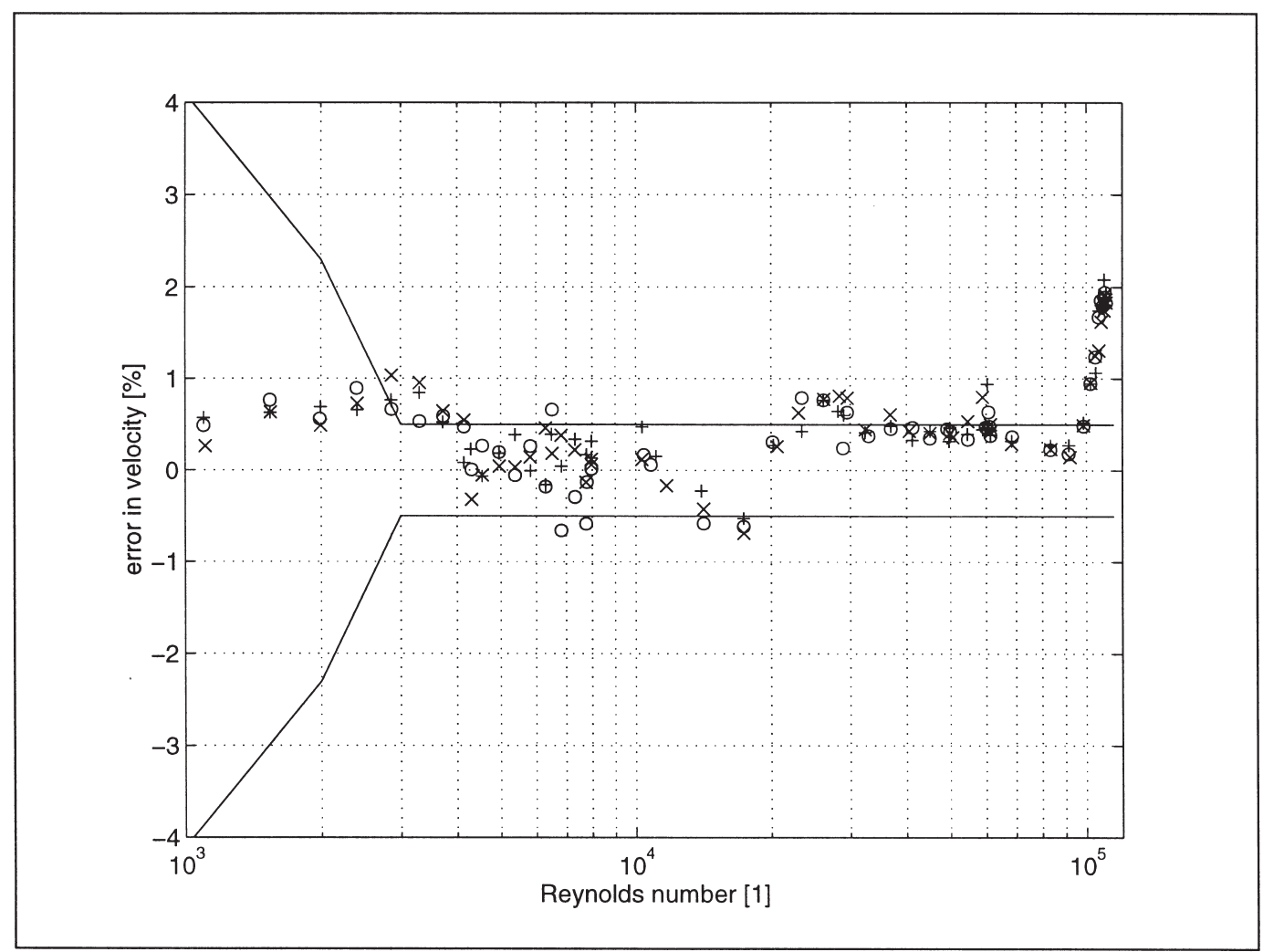

Fig. 12. The percentage error due to the diameter reduction. The solid lines represent the limits confining the six reference measurements with a $95 \%$ confidence level.

smaller, except at the highest flow rates, than those caused by the single and double elbow. The largest error was approximately $2 \%$ and occurred in the range of Reynolds numbers from 100000 to 110000 .

Fig. 13 displays the change in standard deviation in the experiment with the diameter reduction.

As with the error, the distinct change in standard deviation occurs at Reynolds numbers higher than 100000 . Below no clear changes in standard deviation take place. Some but not all of the repeated measurements show a significant increase in the noise level for Reynolds numbers 2000-5000.

The significant errors and increase in noise level occur again at the same flow rate. Where might be a small error in the range over Reynolds numbers 20 000-30 000 without any increase in standard deviation.

\subsection{The pulsating flow experiment}

The fourth disturbance tested was a dynamic installation effect, the pulsating flow. The set-up was the same as in the reference. A rotating valve opened and closed the flow path 4.4 times each second $175 \mathrm{D}, 11025.6 \mathrm{~mm}$ and $6551.2 \mathrm{~mm}$ diameters, in front of the meter [14].

The main frequency of the pulsation is $4.4 \mathrm{~Hz}$ but at Reynolds numbers below 1000 another frequency appears. At Reynolds numbers below 1000 the $6.6 \mathrm{~Hz}$ frequency is dominant. The power spectrum density plots in Fig. 14 exemplifies this. The left plot describes the pulsating flow at Reynolds number 370 and the right at Reynolds number 10 300. At Reynolds number 370 the $6.6 \mathrm{~Hz}$ frequency contains the most power. The 4.4 $\mathrm{Hz}$ frequency is dominant at Reynolds number 10300.

At high flows this arrangement, with the rotating butterfly valve, caused vibrations in the piping. Therefore, a bypass valve was slightly opened at high flows to reduce vibrations. The $(+),(\bigcirc)$ and $(\times)$ marks the data obtained with the bypass valve closed and the $(*)$ marks the data when this valve was partly opened.

The errors caused by the pulsating flow is shown in Figs. 15 and 16.

The measurements with the bypass valve slightly opened showed no significant errors, neither did any of the measurements at Reynolds numbers over 20000. Errors with the magnitude of up to $3 \%$ took place in the range of Reynolds numbers from 3000 to 20000 . As can be seen in Fig. 16, where a Reynolds number down to 10 is shown, large errors, $10-80 \%$, occurred at Reynolds numbers lower than 300 . These very large errors could perhaps be explained by the meter factor curve that is very steep at low flow rates in question. To determine the reason for these large errors further investigations are required.

Here no swirl is present in the flow neither is the flow 


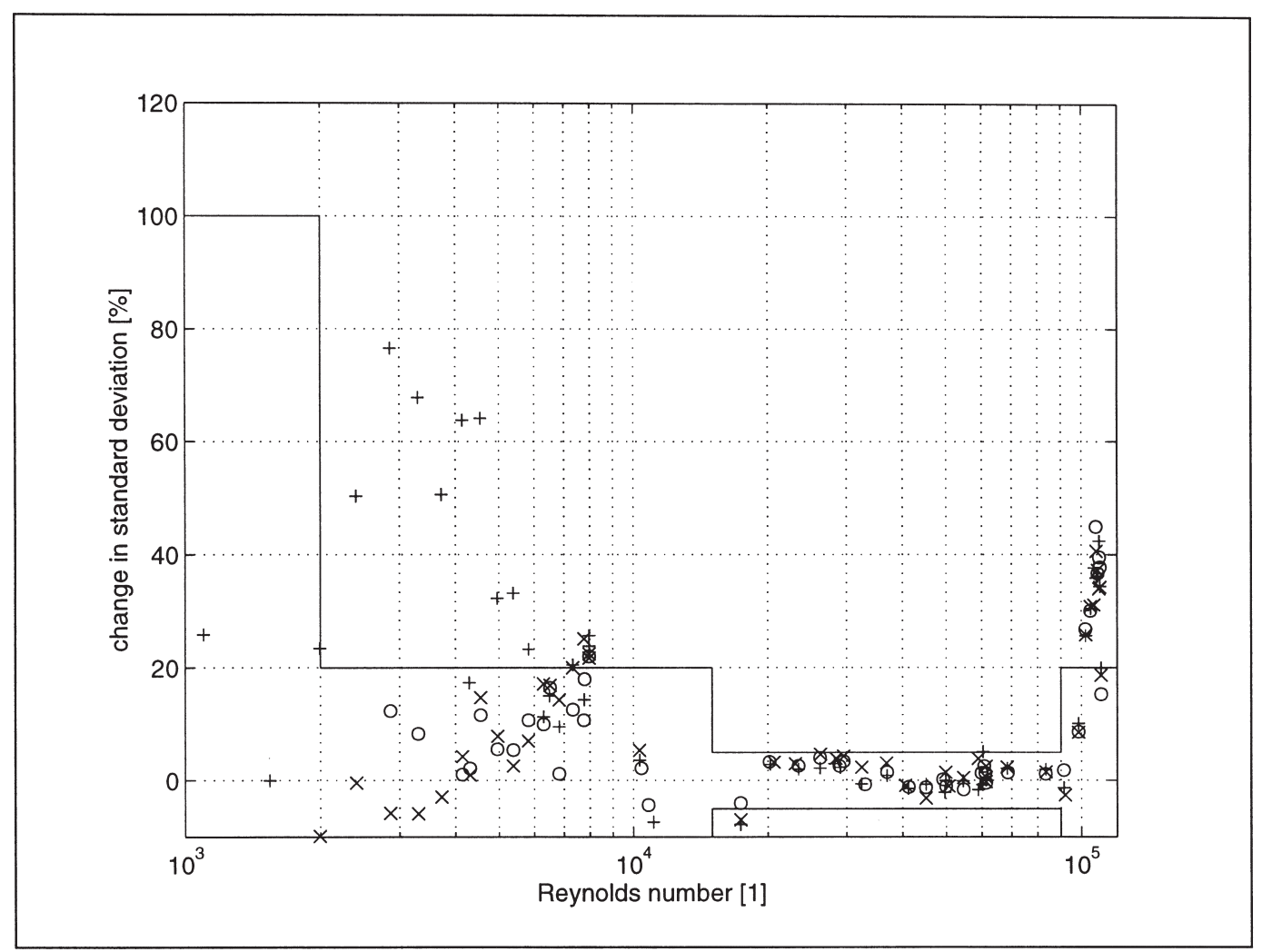

Fig. 13. The change of the standard deviation in per cent $\operatorname{std}_{\text {change }}$ in the diameter reduction experiment. The solid lines represent the limits confining the six reference measurements with a $95 \%$ confidence level.

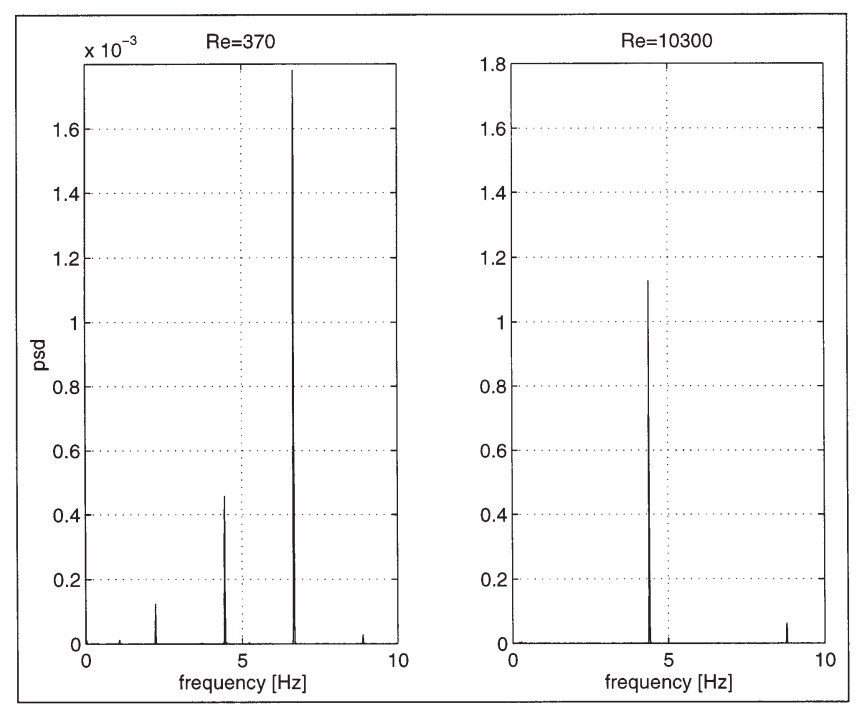

Fig. 14. Two power spectrum density plots of flow meter data from the pulsation experiment. The left plot is based on measurements made at Reynolds number 370 or $0.037 \mathrm{~m} / \mathrm{s}$ and the right at Reynolds number 10300 or $1.033 \mathrm{~m} / \mathrm{s}$.

profile asymmetric. A pulsating flow will cause other effects. At laminar and transient flow the mean flow profile is generally flattened out when a pulsation is present in the flow [15]. This shows as an imaginary high Reynolds number. An ultrasonic flow meter then normally underestimates the flow. A pulsation in fully developed turbulent flows is not likely to affect the performance of the meter [9].

Figs. 17 and 18 display the change in standard deviation in the experiment with the pulsating flow. In Fig. 18 the scale includes Reynolds numbers from 10 to 120000 . 


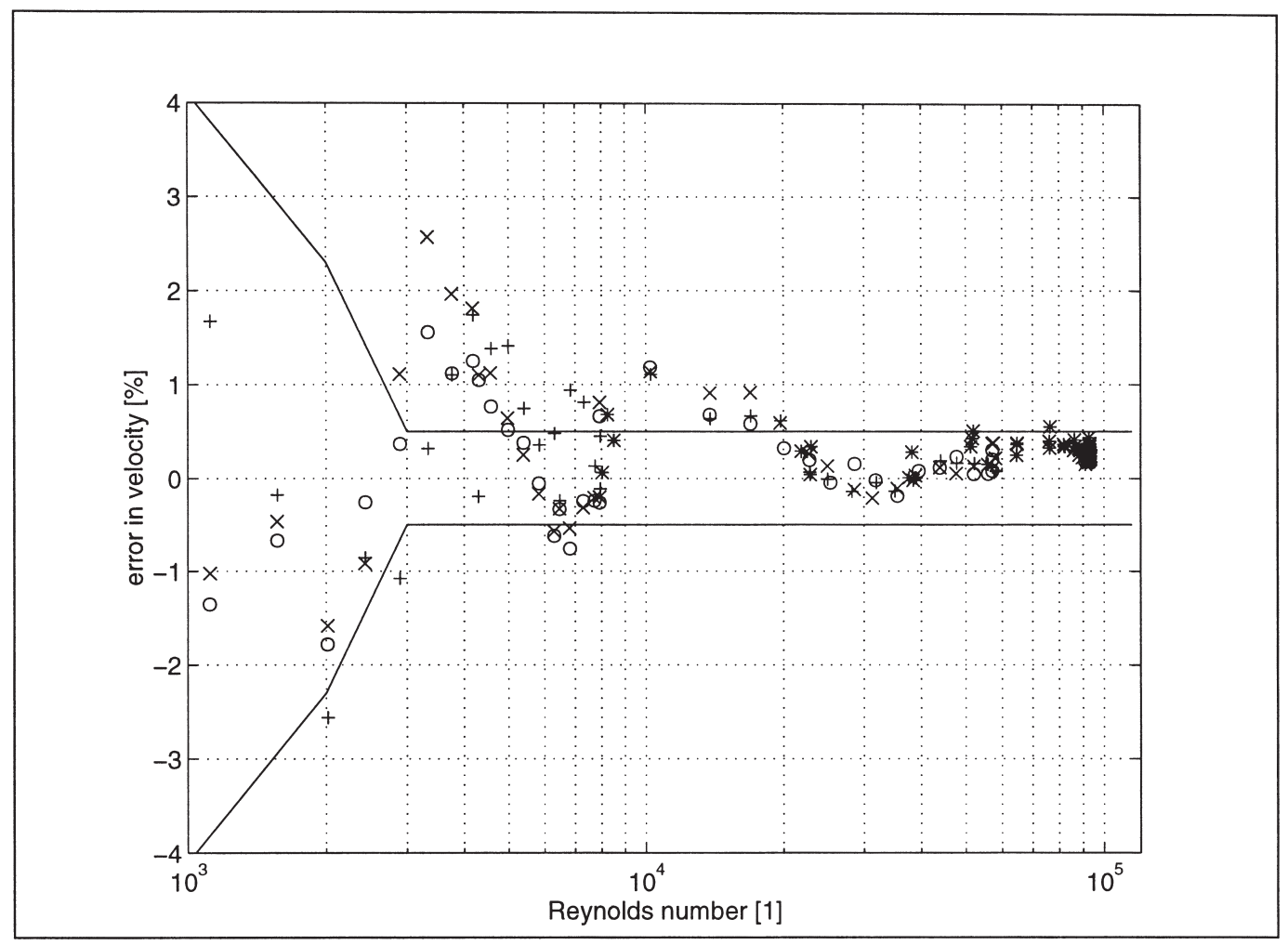

Fig. 15. The percentage error due to the $4.4 \mathrm{~Hz}$ pulsation. The solid lines represent the limits confining the six reference measurements with a $95 \%$ confidence level.

When the bypass valve was opened only the measurement at Reynolds number 8000 shows a significant increase in standard deviation. When the bypass valve was closed the standard deviation on the contrary was highly enlarged compared to the reference case. In the range with Reynolds numbers 20000-60000 the increase was about $20 \%$.

It can he seen that the noise in the data was highly increased at low Reynolds number. At Reynolds numbers from 25 to 20000 the increase was as high as $100-1200 \%$.

In the experiments with different pipe configurations the errors and the increase in noise level occur in most cases at the same flow rate. In this case the pulsating flow causes an increase in standard deviation in the whole flow range over Reynolds numbers 2.5-100 000 except when the bypass valve was open. In the range over Reynolds numbers 300-3000 and 20 000-100 000 there are however not any errors.

\section{Discussion}

The results demonstrate that all installation effects tested give rise to flow metering errors greater than reference conditions as well as significant changes in the noise level calculated as standard deviation. The results are reproducible, at least for the time scale of days. That is how long it took to acquire the data.

1. For Reynolds numbers 3000-110 000, corresponding to a rangeability of 1:40, the six reference measurements were confined within a $\pm 0.5 \%$ limit with a $95 \%$ confidence level. At Reynolds number 500 this limit was $\pm 5 \%$ corresponding to a rangeability of 1:240.

2. In the reference experiment the standard deviation was constant with a magnitude of about $0.3 \mathrm{~mm} / \mathrm{s}$ at laminar flows. Above Reynolds number 2000 the standard deviation increased steadily with increasing Reynolds number.

3. In the single elbow experiment the standard deviation increased at the most with $100 \%$ at Reynolds number 4000. The single elbow caused errors with up to $3 \%$. This largest error also showed up at Reynolds number 4000. At Reynolds number higher than 100000 the increase was about $50 \%$. An error of about $1.5 \%$ also occurred. In most of the flow range there were no or smaller errors.

4. The enlargement of the standard deviation caused by the double elbow was almost identical to the increase generated by the single elbow. The errors were slightly larger, at most about $4 \%$. As in the single elbow case, the errors and the increase in standard deviation showed up in about the same flow ranges. 


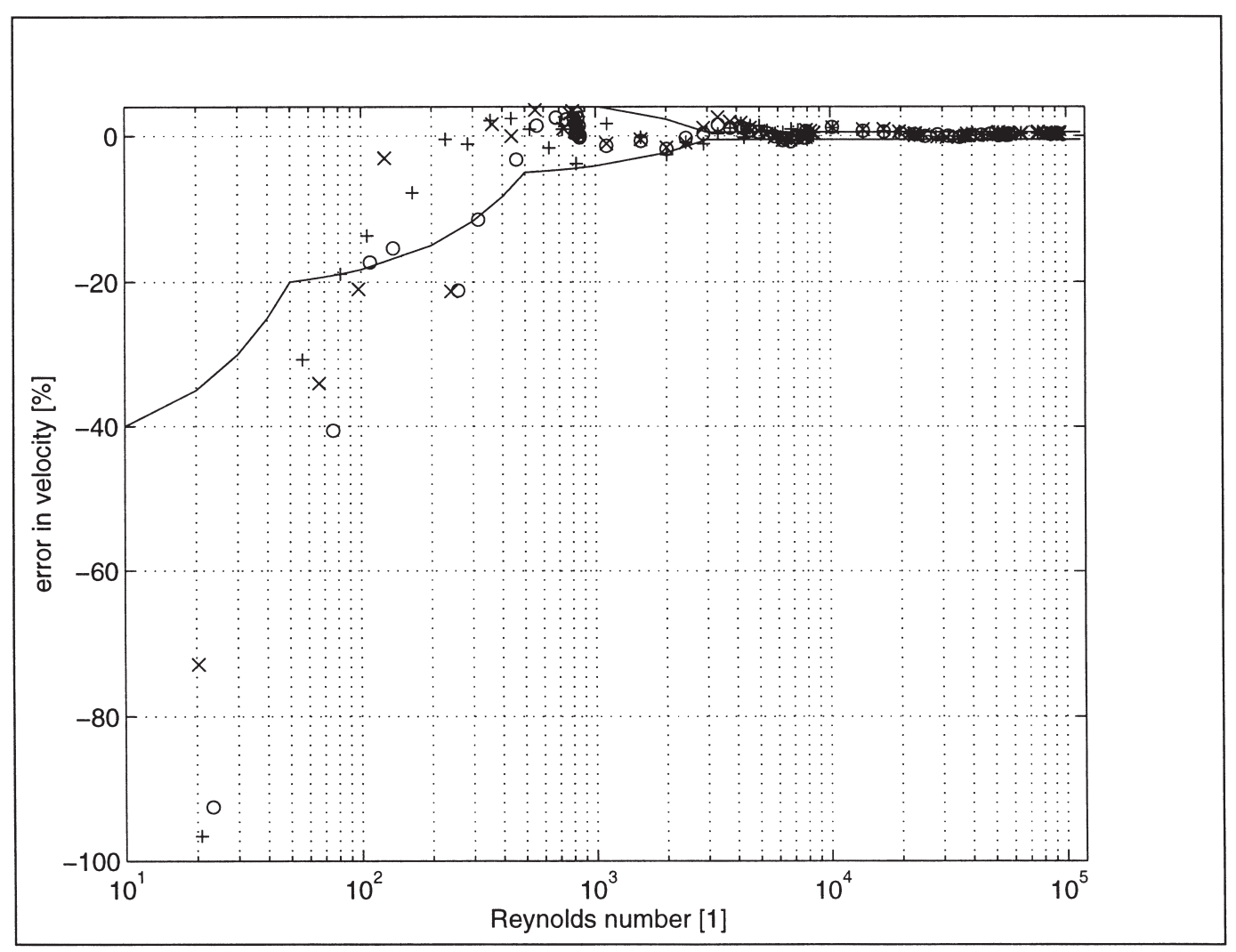

Fig. 16. The percentage error due to the $4.4 \mathrm{~Hz}$ pulsation. The solid lines represent the limits continuing the six reference measurements with a 95\% confidence level.

5. In the diameter reduction experiment the clear change in standard deviation as well as the error took place at Reynolds numbers higher than 100000 . The change in standard deviation was about $40 \%$ and the error slightly higher than $2 \%$.

6. The pulsating flow generated a large increase in the standard deviation compared to the reference case for flow rates with Reynolds numbers lower than 20000 . The enlargement of the noise level was up to $1200 \%$ at Reynolds number 3000. At higher flows with Reynolds numbers from 20000 to 60000 the increase in noise level was about $20 \%$. No significant errors were caused at Reynolds numbers higher than 20000 . The errors were mainly in the range of $1-2 \%$ but at Reynolds numbers below 300 the magnitudes of the errors were as high as $80 \%$.

The results demonstrate that the installation effects tested introduce an error in the flow measurements greater than the error during reference conditions. They also demonstrate that these changes of the installation can be detected from the increase in the noise level of the flow signal compared to the reference case. Further it is clear that where there is a larger error, more than $1 \%$, there is also a very clear increase in standard deviation.

The larger errors mainly occurred in the flow ranges with Reynolds numbers below 40000 or over 100000 . These ranges are also the ranges where the mean meter factor curve in the reference experiment showed up marked slopes. There are perhaps also significant implications for meter design in these results.

The noise level calculated as standard deviation could be interpreted as a measure of the turbulence. And it is very likely that the disturbances do increase the turbulence intensity of the flow. At Reynolds numbers below 2000 , i.e. laminar flow, the signal in the reference experiment only contains the basic noise level. Except for the pulsating flow experiment there were no increase in the noise level due to the disturbances in this flow range. Neither were there any errors in this flow range, except in the case with pulsating flow.

In conclusion, it is clear that the presence of a disturbance could be recognised by simply comparing the magnitude of the standard deviation in the present data with a reference level valid for the measured flow rate. Further it is likely that the marked noise increases are correlated to significant measurement error. It is anticipated that a procedure to detect the introduction of a disturbance and the thereby caused metering errors can be implemented in the flow meter. Thus giving the flow meter a self diagnosing capability. 


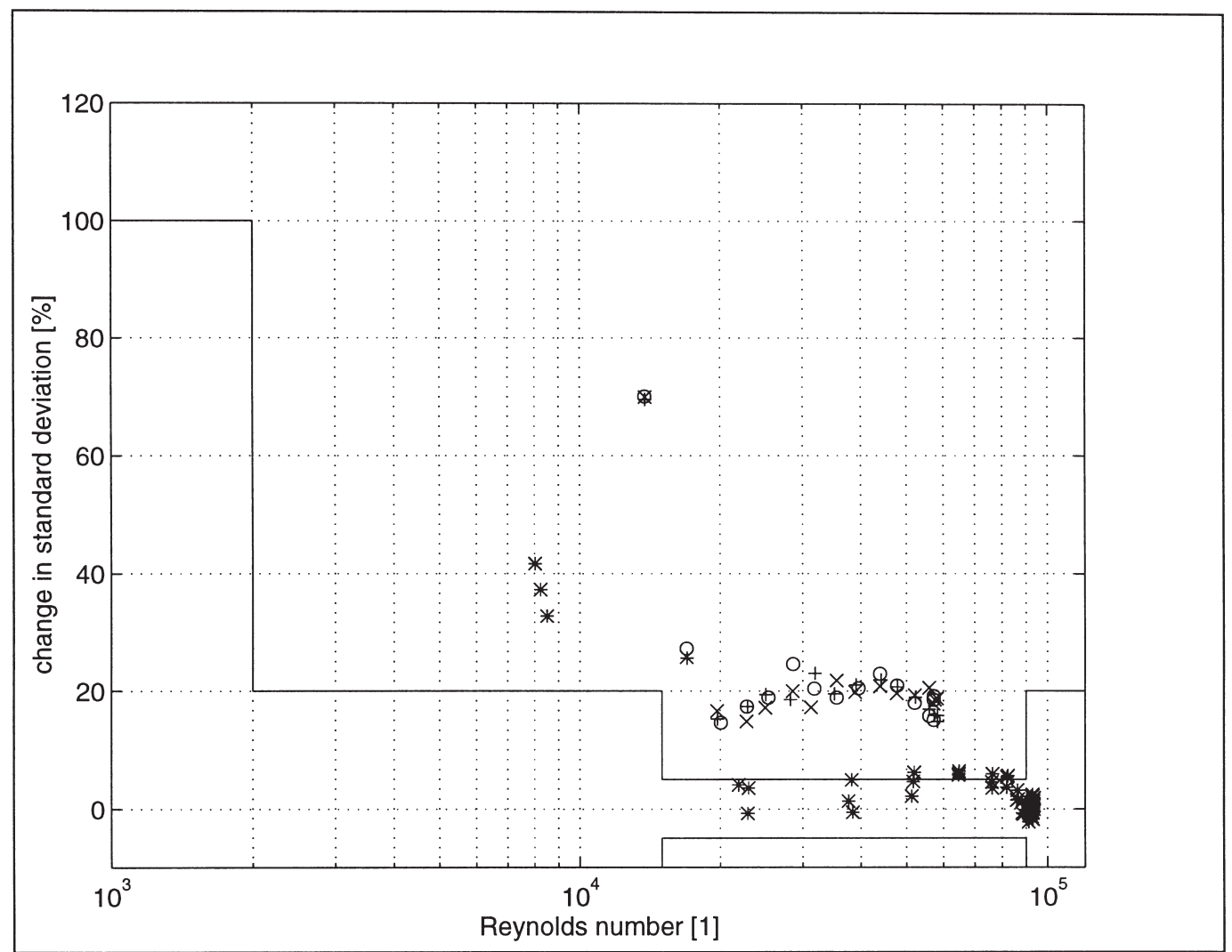

Fig. 17. The change in standard deviation in per cent $\operatorname{std}_{\text {change }}$ due to the $4.4 \mathrm{~Hz}$ pulsation. The solid lines represent the limits confining the six reference measurements with a $95 \%$ confidence level.

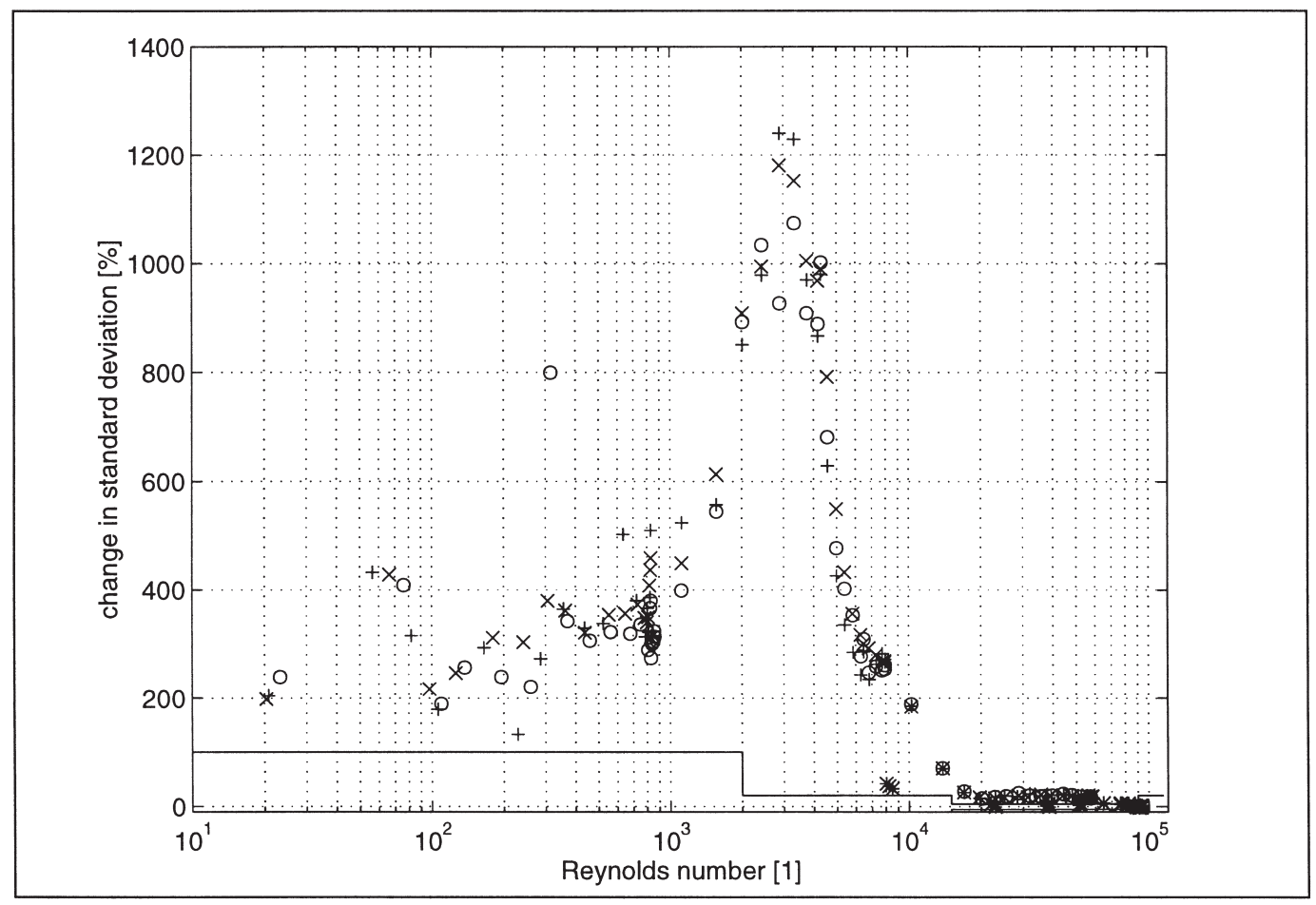

Fig. 18. The change in standard deviation in per cent $\operatorname{std}_{\text {change }}$ due to the $4.4 \mathrm{~Hz}$ pulsation. The solid lines represent the limits confining the six reference measurements with a $95 \%$ confidence level. 


\section{Acknowledgements}

This project is financed under a grant from the Swedish District Heating Association. Mats Lindgren and Daniel Hallgren at D-Flow AB in Luleå placed the ultrasonic flow meter at our disposal.

\section{References}

[1] G.E. Mattingly, T.T. Yeh, Effects of pipe elbows and tube bundles on selected types offlow meters, Flow Measurement and Instrumentation 2 (1989) 4-13.

[2] G. Wendt, B. Mickan, R. Kramer, D. Dopheide, Systematic investigation of pipe flows and installation effects using laser Doppler anemometry - part I. profile measurements downstream of several pipe configurations and flow conditioners, Flow Measurement and Instrumentation 7 (3-4) (1996) 141-149.

[3] B. Mickan, G. Wendt, R. Kramer, D. Dopheide, Systematic investigation of pipe flows and installation effects using laser Doppler anemometry-part II. the effect of disturbed flow profiles on turbine gas meters-a describing empirical model, Flow Measurement and Instrumentation 7 (3/4) (1996) 151-160.

[4] J.E. Heritage, The performance of transit time ultrasonic flow meters under good and disturbed flow conditions, Flow Measurement and Instrumentation 1 (1989) 24-30.

[5] P. Højholt, Installation effects on single and dual beam ultrasonic flow meter, International Conference on Flow Measurement in the mid 80's, National Engineering Laboratory, Scotland, 1985.

[6] E. Håkansson, J. Delsing, Effects of flow disturbances on an ultrasonic gas flow meter, Flow Measurement and Instrumentation 3 (4) (1992) 227-233.

[7] J. Delsing, M. Holm, J. Stang, Computer simulation approach to flow meter installation effects, Proceedings FLOMEKO'93, 1993, pp. $180-187$.

[8] E. Håkansson, J. Delsing, Effects of pulsating flow on an ultrasonic gas flow meter, Flow Measurement and Instrumentation 5 (2) (1994) 93-101.

[9] J. Delsing, Dynamic installation effects on ultrasonic flow meters, Proceedings FLOMEKO'96, 1996, pp. 192-202.

[10] E.H. Higham, R. Fell, A. Ajaya, Signal analysis and intelligent flow meters, Measurement + Control, Flow measurement Special Issue, June 1986.

[11] U.R.C. Nilsson, In situ detection of inaccurate gas flow meters using a fingerprint technique, Licentiate Thesis, ISSN 1402-1757, ISRN LTU-LIC-1997/37-SE, 1997.

[12] J. Delsing, Viscosity effects in sing-around type flow meters, Proceedings International Conference on Industrial Flow Measurement Onshore \& Offshore, IBC London, September, 1987.

[13] C. Carlander, Installation effects and implications for self diagnostics for an ultrasonic flow meter, Licentiate Thesis 1998:28, Luleå University of Technology, ISSN 1402-1757, ISRN LTULIC-1998/28, 1998.

[14] C. Carlander, J. Delsing, Installation effects on an ultrasonic flow meter, Proceedings FLOMEKO'98, 1998.

[15] D.C. Mizushina, T. Maruyama, Y. Shiozaki, Pulsating turbulent flow in a tube, Journal of Chemical Engineering of Japan 6 (6) (1973) 487-494. 